\title{
Análise das experiências educacionais dos estudantes com simulações em realidade aumentada móvel para o desenvolvimento do conhecimento em Física
}

\author{
Fabrício Herpich ${ }^{1}$, Liane Margarida Rockenbach Tarouco ${ }^{1}$ \\ ${ }^{1}$ Programa de Pós-Graduação Informática na Educação (PPGIE) \\ Centro Interdisciplinar de Novas Tecnologias na Educação (CINTED) \\ Universidade Federal do Rio Grande do Sul (UFRGS) \\ Porto Alegre - RS - Brazil \\ fabricio.herpichegmail.com, lianeepenta.ufrgs.br
}

\begin{abstract}
The use of information and communication technologies has been presented as a complementary alternative to the process of teaching and learning Physics, especially with the advent of mobile devices and the availability of multimedia resources, promoting access and interaction with educational experiences to users. In this perspective, this article aims to investigate the impact that experiences with increased educational resources on mobile devices have for the development of knowledge in Physics of its users. To achieve this goal, a quasi-experimental study with 208 participants was conducted, which allowed us to demonstrate the effect that the interactions provided with this technology have on the development of knowledge in Physics.
\end{abstract}

Resumo. $O$ uso de tecnologias de informação e comunicação tem se apresentado como alternativa complementar ao processo de ensino e aprendizagem de Física, principalmente com o advento dos dispositivos móveis e a disponibilidade de recursos multimídia, promovendo o acesso e a interação com experiências educacionais aos usuários. Nesta perspectiva, este artigo tem como objetivo investigar o impacto que as experiências com recursos educacionais aumentados em dispositivos móveis possuem para o desenvolvimento do conhecimento em Física de seus usuários. Para alcançar esse objetivo, um estudo quasi-experimental com 208 participantes foi realizado, o que possibilitou demonstrar o efeito que as interações proporcionadas com esta tecnologia possuem para o desenvolvimento dos conhecimentos em Física.

\section{Introdução}

Ensinar e aprender conteúdos referentes às Ciências Físicas tem se mostrado uma tarefa difícil nos últimos anos, especialmente no contexto brasileiro. Nesta perspectiva, os dados do PISA demonstram que o Brasil tem apresentado dificuldades no desenvolvimento do ensino de conteúdos relacionados à Ciências, sendo que dentre 70 países participantes (média de pontos 493), a posição do Brasil (média de pontos 401) durante a última avaliação foi $63^{\circ}$, ficando à frente apenas do Peru (média de pontos 397), Líbano (média de pontos 386), Tunísia (média de pontos 386), Macedônia (média de pontos 384), Kosovo (média de pontos 378), Argélia (média de pontos 376) e República Dominicana (média de pontos 332) [OECD 2016]. 
VIII Congresso Brasileiro de Informática na Educação (CBIE 2019)

Anais dos Workshops do VIII Congresso Brasileiro de Informática na Educação (WCBIE 2019)

Pesquisas recentes apontam que as áreas das Ciências, Tecnologia, Engenharia e Matemática (STEM), demandam habilidades específicas dos estudantes, tais como a capacidade espacial, as habilidades práticas, a compreensão conceitual e a investigação científica, para o pleno processo de ensino e aprendizagem [Ibáñez and Delgado-Kloos 2018]. Dentre as habilidades demandadas, uma característica que estas áreas de conhecimento têm em comum, consiste na exigência da habilidade imaginativa dos estudantes para compreender as leis da Física e as reações Químicas, em virtude do fato de que essas manifestações muitas vezes são abstratas e, assim, não podem ser visualizadas pelos alunos, ou então são microscópicas, sendo apenas visualizáveis com o uso de equipamentos de ampliação científicos.

Entretanto, os professores nem sempre têm condições de oferecer atividades práticas devido à falta de laboratórios nas escolas e, além disso, vários experimentos não são capazes de expor completamente os comportamentos naturais que estão ocorrendo uma vez que certos fenômenos não são visíveis ao olho humano, como a eletricidade, o magnetismo e o calor, por exemplo. Alguns podem até mesmo acarretar em riscos ambientais ou pessoais (como a radioatividade) e, portanto, não podem ou não devem ser realizados sem as devidas medidas de segurança, o que impede que sejam realizados na maioria das escolas e até mesmo em algumas universidades [Herpich et al. 2018].

Por outro lado, a introdução de modernas tecnologias da informação e comunicação, juntamente com as formas contemporâneas de educação, conforme [Daineko et al. 2017], estão aumentando significativamente a experiência de ensino e melhorando a qualidade da educação. Os autores descrevem que soluções baseadas em laboratórios virtuais, poderiam ser integrados aos currículos existentes, a fim de ajudar os estudantes a dominar determinadas disciplinas de Ciências experimentais.

No contexto de laboratórios virtuais, uma tecnologia com potencial para fins pedagógicos consiste na realidade aumentada (RA), uma vez que esta tecnologia permite incorporar uma vasta gama de recursos multimídia, além de oferecer a capacidade de portabilidade entre diferentes dispositivos tecnológicos. No cenário educacional a RA tem demonstrado avanços significativos, incentivados pelo crescimento exponencial dos dispositivos móveis nos últimos anos, recebendo destaque em importantes relatórios internacionais, e.g. New Media Consortium (NMC) [Becker et al. 2017] e Organização para a Cooperação e Desenvolvimento Econômico (OECD) [Leopold et al. 2018].

Nesta perspetiva, este artigo tem como objetivo proporcionar aos estudantes experiências educacionais através do uso da realidade aumentada em dispositivos móveis, a fim de avaliar a capacidade que as interações proporcionadas com esta tecnologia possuem para o desenvolvimento do conhecimento em Física de seus usuários. Na expectativa de alcançar este objetivo, definiu-se a seguinte questão de pesquisa:

- Questão de Pesquisa: Experiências educacionais de estudantes com simulações em realidade aumentada móvel são capazes de contribuir para o desenvolvimento do conhecimento em Física?

\section{Realidade Aumentada na Educação}

A RA consiste na integração de recursos multimídia com elementos físicos do mundo real, em que os elementos gráficos criados por computador são apresentados no dis- 
VIII Congresso Brasileiro de Informática na Educação (CBIE 2019)

Anais dos Workshops do VIII Congresso Brasileiro de Informática na Educação (WCBIE 2019)

positivo tecnológico do usuário, simultaneamente com os elementos do ambiente real [Herpich et al. 2019].

No cenário educacional, através da RA e dos seus recursos, é possível ampliar os canais de interação dos usuários com conteúdos educacionais e ensejar maiores oportunidades de aprendizagem. Outros benefícios possibilitados pela RA consistem no alcance de níveis mais elevados de motivação e engajamento dos usuários; a visualização 3D de objetos virtuais interpostos aos reais; a visualização em escala de fenômenos que não são perceptíveis no mundo real e a partir de diferentes perspectivas ou ângulos; características que auxiliam os usuários na assimilação de conceitos abstratos e complexos, facilitando a compreensão sobre um determinado conteúdo educacional [Herpich et al. 2019]. Adicionalmente, fornece experiências visuais e interativas únicas, as quais dão suporte para a compreensão de fenômenos complexos, que combinam informações reais e virtuais e ajudam a comunicar problemas abstratos aos estudantes [Billinghurst and Dünser 2012].

A respeito do potencial pedagógico, de acordo com [Cai et al. 2014] a RA pode apresentar recursos multimídia em escala, transformando os recursos didáticos tradicionais (e.g. imagens e livros didáticos em 2D). Através desta estratégia de relacionar o conteúdo virtual de aprendizagem com os objetos reais, a literatura tem apresentado que as soluções educacionais em RA podem contribuir de modo significativo na abstração dos estudantes em conteúdos de Física. Além disso, [Nincarean et al. 2013] acrescentam que a eficácia da RA pode ser ampliada quando combinada com outras tecnologias, tais como dispositivos móveis, incorporando os recursos característicos destas tecnologias móveis (e.g. portabilidade, interatividade social, conectividade, sensibilidade ao contexto e individualidade) com os recursos da RA, aspecto que os autores destacam que fomenta $o$ surgimento de um novo conceito, denominado de Realidade Aumentada Móvel.

\section{Metodologia}

Os procedimentos metodológicos implementados para a realização desta pesquisa são descritos nas próximas seções:

\subsection{AVATAR - Ambiente Virtual de Aprendizagem e Trabalho Acadêmico Remoto}

Os recursos em RA utilizados durante os testes desta pesquisa são oriundos do aplicativo avatAR UFRGS [Herpich 2019], que se trata de um módulo para dispositivos móveis do Projeto AVATAR [AVATAR 2019]. O aplicativo avatAR UFRGS é uma tecnologia educacional que proporciona aos usuários o acesso a simulações educacionais desenvolvidas em RA, com o objetivo de implementar formas de oportunizar a aprendizagem de Física ao usuário, por meio de recursos que enfatizam a interação com diferentes materiais pedagógicos. Ao acessar o aplicativo, os usuários têm acesso a diversos recursos educacionais, em que podem visualizar fenômenos físicos micro e macroscópicos, por vezes invisíveis a sua percepção, e interagir com diversos recursos multimídia, e.g. imagens, vídeos, objetos 3D e simulações.

\subsection{Protocolo de testes executados}

$\mathrm{Na}$ expectativa de alcançar o objetivo definido para este artigo, o design de pesquisa praticado foi o quasi-experimental, por disponibilizar métodos investigativos que melhor se enquadram aos testes planejados com os participantes desta pesquisa e com as amostras 
VIII Congresso Brasileiro de Informática na Educação (CBIE 2019)

Anais dos Workshops do VIII Congresso Brasileiro de Informática na Educação (WCBIE 2019)

de dados coletados, assim como por proporcionar técnicas capazes de contribuir no desempenho desta investigação, no que tange responder à seguinte questão de pesquisa:

- Questão de Pesquisa: Experiências educacionais de estudantes com simulações em realidade aumentada móvel são capazes de contribuir para o desenvolvimento do conhecimento em Física?

Para responder à questão de pesquisa elencada acima, elaborou-se juntamente ao professor o Protocolo de Testes (Figura 1), para contrastar as atividades a serem desempenhadas pelos participantes. É possível observar que os participantes foram distribuídos em dois Grupos, denominados como Grupo Controle (GC) e Grupo Experimental (GE), e suas atividades foram descritas nas seguintes etapas: 1) Avaliação inicial sobre o conteúdo da disciplina (Pré-Teste), para identificar o nível de conhecimento de cada grupo; 2) A representação das aulas, atividades e conteúdos inerentes a sala de aula, da mesma forma para ambos os Grupos; 3) Uso de materiais tradicionalmente empregados no processo de ensino e aprendizagem da escola, tais como livros e exercícios, para ambos os Grupos, sendo que adicionalmente, o GE também faz o uso do aplicativo de RA; por fim, 4) Avaliação final sobre o conteúdo da disciplina (Pós-Teste), com o objetivo de identificar se houve avanço no nível de conhecimento de cada grupo em relação ao pré-teste.

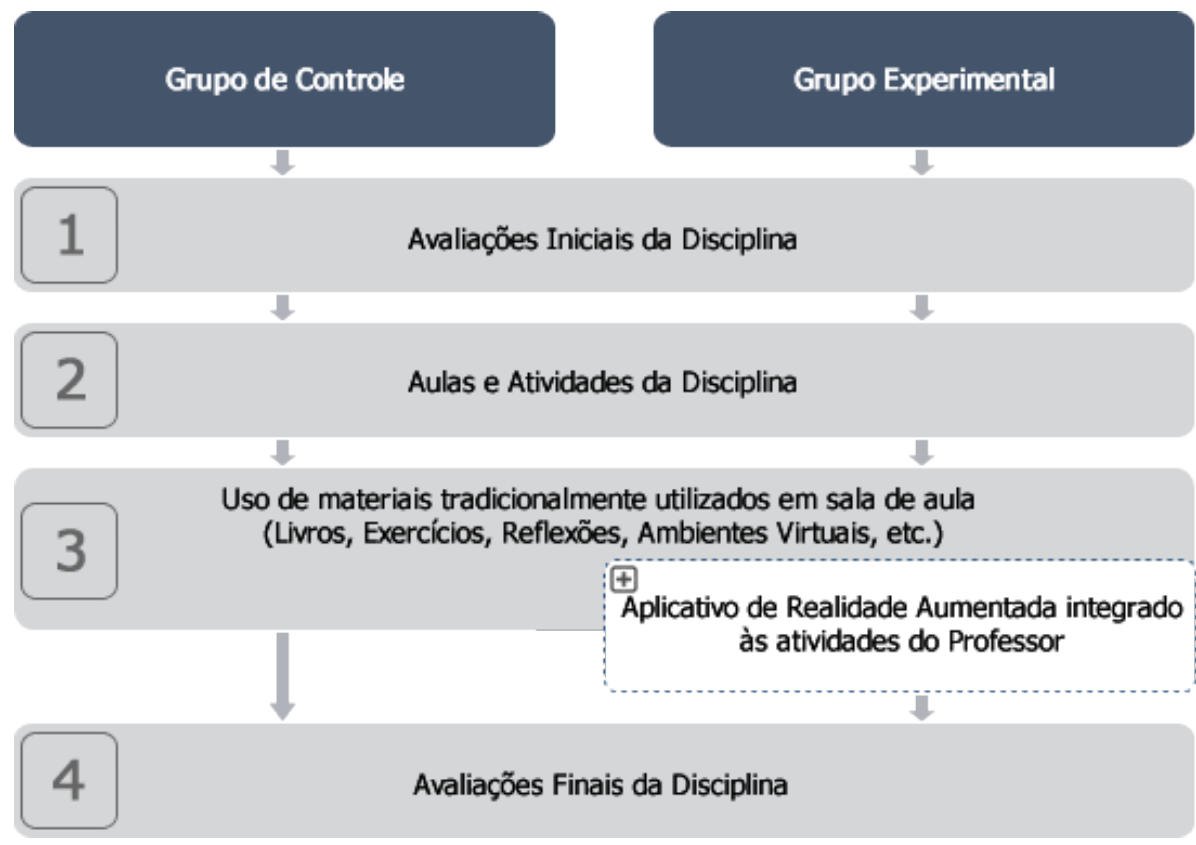

Figura 1. Protocolo de Testes

Em relação ao tempo de duração do ciclo de atividades descritas no Protocolo de Testes (Figura 1), a média de interação dos participantes com o aplicativo de RA foi de 95 dias. Encontros foram realizados entre os pesquisadores responsáveis por esta pesquisa e as turmas que participaram da atividade, com o objetivo de promover a assistência aos participantes em questões envolvendo o uso do aplicativo, acesso aos seus recursos e funcionalidades, apresentando novas simulações e sanando eventuais dúvidas acerca da interação com os recursos educacionais aumentados (durante 45 minutos e periodicidade quinzenal). O professor responsável pela disciplina acompanhou todos os encontros reali- 
VIII Congresso Brasileiro de Informática na Educação (CBIE 2019)

Anais dos Workshops do VIII Congresso Brasileiro de Informática na Educação (WCBIE 2019)

zados entre o pesquisador e as turmas, atuando como mediador e auxiliando na resolução das dúvidas que surgiam sobre o conteúdo específico da disciplina.

\subsection{Dados demográficos dos participantes}

Participaram desta pesquisa 208 sujeitos, os quais foram distribuídos em dois grupos: Grupo Controle e Grupo Experimental. No GE foram designados aqueles participantes que interagiram com o aplicativo de RA (53,8\% dos participantes), já os participantes que não interagiram com o aplicativo foram considerados pertencentes ao GC (46,2\% dos participantes). Os sujeitos estão distribuídos em diferentes níveis na estrutura da educação básica: duas turmas do $9^{\circ}$ ano do Ensino Fundamental (E.F.) (turmas A e B), duas turmas do $1^{\circ}$ ano do Ensino Médio (E.M.) (turmas 101 e 102), uma turma do $2^{\circ}$ ano do E.M. (turma 201), e outras três turmas do $3^{\circ}$ ano do E.M. (turmas 301, 302, 303).

A respeito da distribuição de gêneros entre os participantes desta pesquisa, 57,2\% dos participantes correspondem ao gênero masculino e $42,8 \%$ correspondem ao gênero feminino (totalizando 89 participantes). De modo mais específico, $56 \%$ dos participantes pertencem ao GE (52 participantes do gênero feminino e 60 participantes do gênero masculino) e $44 \%$ ao GC (37 do gênero feminino e 59 participantes do gênero masculino). Em se tratando da faixa etária da população, os dados demográficos demonstram que $7,2 \%$ pertencem à faixa etária de 14 anos, 27,9\% compreendem a faixa etária de 15 anos, 26,9\% correspondem a faixa etária de 16 anos, 30,3\% estão na faixa etária de 17 anos, $7,2 \%$ pertencem à faixa etária de 18 anos, e, por fim, $0,5 \%$ corresponde a faixa etária de 19 anos (apenas 1 participante).

\subsection{Instrumento de coleta de dados}

Para a coleta de dados do nível de conhecimento dos participantes em Física, foi elaborado um instrumento que os participantes responderam, o qual adotou a sistematização de PréTeste e Pós-Teste. O instrumento foi elaborado pelo professor responsável da disciplina e aplicado num intervalo médio de 95 dias entre o Pré-Teste e Pós-Teste. No momento do Pré-Teste, nenhum participante havia interagido com os recursos do aplicativo de RA utilizado nessa pesquisa ou com qualquer outro recurso educacional aumentado.

\subsubsection{Análise dos dados}

Na expectativa de atender ao objetivo desta pesquisa com embasamento em análises estatísticas, os dados coletados durante a execução do Protocolo de Testes (Figura 1), foram organizados na forma de um banco de dados descrevendo as variáveis de interesse desta pesquisa com vistas a evidenciar a existência de resultados estatisticamente significativos. Foram definidas duas hipótese para a questão de pesquisa, sendo a primeira denominada de Hipótese Nula (H0) e a segunda de Hipótese Alternativa (H1), busca-se através da análise estatística, aceitar ou rejeitar uma das hipóteses:

- H0: não há diferenças significativas no desempenho mediano da aprendizagem entre o GE (experiências educacionais em RA) e GC (experiências tradicionais de sala de aula).

- H1: houve diferenças significativas no desempenho mediano da aprendizagem entre o GE (experiências educacionais em RA) e GC (experiências tradicionais de sala de aula). 
VIII Congresso Brasileiro de Informática na Educação (CBIE 2019)

Anais dos Workshops do VIII Congresso Brasileiro de Informática na Educação (WCBIE 2019)

Foram desempenhados os testes Wilcoxon e Kruskal-Wallis, com as amostras pareadas, dado que os mesmos participantes foram comparados tanto no Pré-teste como no Pós-teste. Também foi executado o teste para significação de correlação de Spearman com as amostras do GE, para a análise do desempenho dos participantes nas avaliações do nível de conhecimento do conteúdo em relação as interações com o aplicativo de RA. O intervalo de confiança para a execução dos testes estatísticos foi definido em 95\%, que representa o nível de significância de 0,05 .

\section{Análise dos Resultados}

\subsection{Análise do desempenho dos participantes entre grupos de mesma série}

A fim de analisar o desempenho em Física dos participantes em cada grupo observado durante esta pesquisa, testes estatísticos foram realizados para evidenciar se houve diferença significativa entre o desempenho de cada grupo para cada série, assim como se houve diferença significativa entre o resultado do Pré-Teste e Pós-Teste que determinou o nível de conhecimento em Física.

A primeira análise realizada verificou se as Turmas do $9^{\circ}$ ano do E.F. demonstraram diferença estatisticamente significativa entre a mediana dos participantes do GC para o GE durante o Pré-Teste que determinou o nível de conhecimento em Física dos participantes (Tabela 1). Tal análise possibilitou evidenciar que não houve diferença estatística significativa, dado que o p-valor foi de 0.5868 . Nesta perspectiva, a rejeição da hipótese nula retrata um resultado positivo para esta investigação, dado que apresenta evidências da uniformidade entre os grupos antes mesmo do início dos testes, aspecto que representa a homogeneidade dos grupos antes da realização das atividades com os recursos educacionais aumentados. No Pós-Teste realizado com o $9^{\circ}$ ano do E.F., foi possível verificar que houve uma melhora significativa na mediana relativa ao desempenho do GE em relação ao GC, evidência que demonstra diferença estatística (p-valor 0.005449) (Tabela 1). A evidência encontrada é suficiente para rejeitar a hipótese nula e aceitar a hipótese alternativa, indício de que os participantes que utilizaram os recursos educacionais aumentados obtiveram melhor performance na mediana referente ao nível de conhecimento em Física em relação aos participantes que não utilizaram os recursos educacionais aumentados. Além disso, também foi possível verificar que apenas o GE obteve diferença estatisticamente significativa entre o Pré-Teste e o Pós-Teste ( $p$-valor $<0,0001184$ ), perspectiva que corrobora para a inferência de que a melhora na performance do conhecimento de Física não ocorreu por acaso, mas sim em virtude do uso dos recursos educacionais em RA.

Outro teste estatístico foi executado com os resultados das Turmas do $1^{\circ}$ ano do E.M., em que não foi possível verificar a ocorrência de diferença significativa entre a mediana de desempenho dos participantes do GC e o GE durante o Pré-Teste de conhecimento em Física (p-valor 0.5657), resultado positivo que demonstra que os grupos eram homogêneos no início dos testes (Tabela 1). Entretanto, no Pós-Teste também não houve diferença significativa entre os grupos ( $\mathrm{p}$-valor 0.4852).

Embora tenha sido verificado aumento na mediana dos participantes do GE em relação ao GC, que se trata de um importante resultado, a diferença observada não foi suficiente para comprovar estatisticamente que os recursos educacionais aumentados contribuíram para o desenvolvimento da performance em Física. Entretanto, foi constatado resultados positivos para ambos os grupos, quando verificado a melhora no desempenho 
VIII Congresso Brasileiro de Informática na Educação (CBIE 2019)

Anais dos Workshops do VIII Congresso Brasileiro de Informática na Educação (WCBIE 2019)

9o ano do E.F. - Turmas 9A / 9B

\begin{tabular}{|c|c|c|c|}
\hline Avaliação & Controle & Experimental & p-valor \\
\hline Pré-Teste (CF*) & 6,8 & 6,7 & 0.5868 \\
\hline Pós-Teste (CF) & 7,6 & 8,2 & 0.005449 \\
\hline p-valor & 0.2802 & 0.0001184 & \\
\hline \multicolumn{4}{|c|}{ 1 ano do E.M. - Turmas 101/ 102} \\
\hline Avaliação & Controle & Experimental & p-valor \\
\hline Pré-Teste (CF) & 5,3 & 4,85 & 0.5657 \\
\hline Pós-Teste (CF) & 6 & 7 & 0.4852 \\
\hline p-valor & 0.002344 & $<0,001$ & \\
\hline \multicolumn{4}{|c|}{ 2ㅇ ano do E.M. - Turma 201} \\
\hline Avaliação & Controle & Experimental & p-valor \\
\hline Pré-Teste (CF) & 6,7 & 5,5 & 0.1126 \\
\hline Pós-Teste (CF) & 6,7 & 5,85 & 0.275 \\
\hline p-valor & 0.777 & 0.4504 & \\
\hline \multicolumn{4}{|c|}{ 3 ano do E.M. - Turmas $301 / 302$ / 303} \\
\hline Avaliação & Controle & Experimental & p-valor \\
\hline Pré-Teste (CF) & 6,7 & 6,7 & 0.7637 \\
\hline Pós-Teste (CF) & 7,85 & 7,85 & 0.8081 \\
\hline p-valor & 0.0005651 & 0.0002748 & \\
\hline
\end{tabular}

${ }^{*} \mathrm{CF} \rightarrow$ Conhecimento em Física

Tabela 1. Comparações entre Grupos da mesma série em relação ao desempenho de Física

entre o Pré-Teste e o Pós-Teste de conhecimento em Física, dado que tanto o GC (p-valor 0.002344 ) como o GE ( $\mathrm{p}$-valor $<0,001$ ) apresentaram diferença estatisticamente significativa. Padrão que também ocorreu para os resultados das Turmas do $3^{\circ}$ ano do E.M. Já os resultados do desempenho da Turma do $2^{\circ}$ ano do E.M., não foi verificada diferença em nenhuma das análises estatísticas realizadas.

\subsection{Análise global do desempenho dos participantes}

Na expectativa de comparar o desempenho global entre o GC e o GE, testes estatísticos foram conduzidos para comparar ambos os grupos englobando todas as amostras apresentadas nas análises anteriores (Tabela 2). A partir da análise dos resultados, foi possível evidenciar que o desempenho de ambos os grupos na primeira avaliação de conhecimento em Física não apresentou diferença estatística significativa (p-valor 0.1366), resultado positivo e que reforça o fato dos grupos serem homogêneos antes mesmo da realização desta pesquisa.

Através da análise dos resultados coletados da segunda avaliação que determinou o nível de conhecimento em Física dos participantes, foi possível constatar que não houve diferença significativa entre as performances do GC em relação ao GE (p-valor 0.7839), resultado negativo constatado também nas medianas do GE, que não apresentaram melhora substancial capaz de comprovar estatisticamente a eficácia dos recursos educacio- 
VIII Congresso Brasileiro de Informática na Educação (CBIE 2019)

Anais dos Workshops do VIII Congresso Brasileiro de Informática na Educação (WCBIE 2019)

\begin{tabular}{lcccc}
\hline Total & $\mathbf{n}$ & Pré-Teste (CF*) & Pós-Teste (CF) & p-valor \\
\hline Controle & 96 & 6,4 & 7,35 & $<0,001$ \\
Experimental & 112 & 6 & 7 & $<0,001$ \\
\hline p-valor & 0.1366 & 0.7839 & \\
\hline${ }^{*}$ CF $\rightarrow$ Conhecimento em Física
\end{tabular}

Tabela 2. Comparação geral do GC com o GE

nais aumentados. Por outro lado, foi possível observar que ambos os grupos obtiveram melhor desempenho entre o Pré-Teste e o Pós-Teste, perspectiva que apresentou diferença estatística significativa ( $\mathrm{p}$-valor $<0,001$ ) em ambos os casos. Nesta perspectiva, pode-se observar que tanto o GE como o GC obtiveram um desenvolvimento estatisticamente significativo nos conhecimentos de Física entre o período do Pré-Teste e o Pós-Teste. Porém, o desenvolvimento observado para o GE não foi o suficiente para se afirmar estatisticamente que os recursos educacionais em RA foram capazes de melhorar o aprendizado em Física. Em vista do resultado, considera-se a continuidade da pesquisa, a fim de possibilitar um maior intervalo de tempo para a experiência e o contato dos participantes com os recursos educacionais aumentados, para que seja possível estabelecer se houve ganhos significativos de aprendizagem.

\subsection{Interação dos participantes com os recursos educacionais em RA}

Para identificar o quanto às interações dos participantes com os recursos educacionais aumentados influenciaram na performance individual nas avaliações de conhecimento em Física, foi realizada a análise de dispersão para representar graficamente a relação entre o desempenho dos participantes e as suas interações. Para a elaboração deste gráfico foram consideradas apenas as amostras do GE, visto que foram os únicos participantes a utilizarem os recursos educacionais aumentados. O valor utilizado como referência para o desempenho (Eixo Y) foi a diferença dos resultados do participante entre o Pré-Teste e o Pós-Teste do conhecimento em Física. Em relação ao número de interações para cada participante (Eixo X), o valor de referência utilizado foi a quantidade de interações coletadas no log (registro) do aplicativo de RA de cada participante (Figura 2).

Através da representação gráfica apresentada acima (Figura 2) é possível observar uma tendência indicando que quanto maior for a quantidade de interações do usuário, maior será o seu desempenho em Física, porém não se pode estimar o quanto as interações foram determinantes no desempenho de Física. Para determinar o nível da relação entre as variáveis de desempenho em Física e número de interações, foi utilizado a medida de associação denominada coeficiente de correlação de Spearman. O coeficiente obtido foi de 0,24403 , resultado que evidencia a existência a associação entre as variáveis, com significância estatística (p-valor 0.009517). Este resultado demonstra que o desempenho em Física é influenciado positivamente pelas interações do participante.

\section{Conclusão}

A reconhecida dificuldade dos estudantes em construir o conhecimento sobre Física é tema de diferentes pesquisas, publicadas ao longo dos anos. Acerca deste tema, importantes evidências foram apresentadas no relatório sobre os resultados do PISA a nível 
VIII Congresso Brasileiro de Informática na Educação (CBIE 2019)

Anais dos Workshops do VIII Congresso Brasileiro de Informática na Educação (WCBIE 2019)

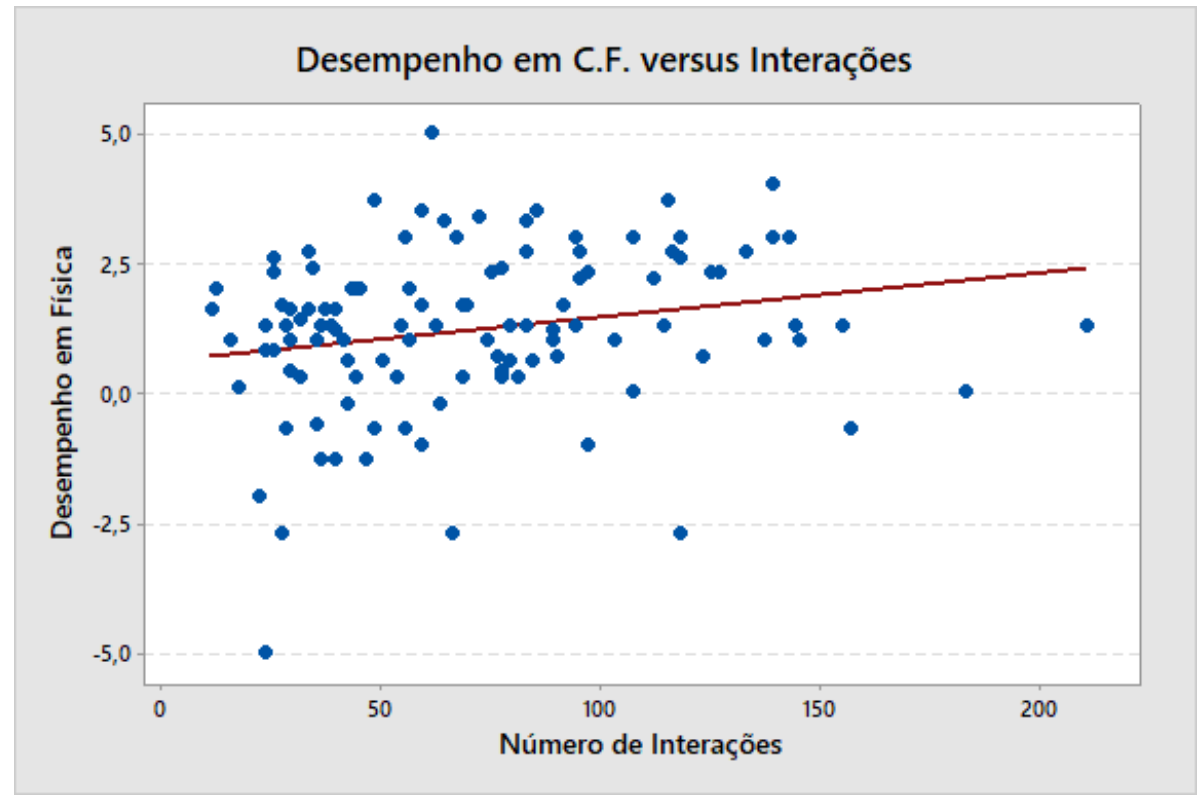

Figura 2. Gráfico de dispersão do desempenho em Física e das interações

mundial [OECD 2016], as quais destacam a desigualdade no desenvolvimento educacional nas áreas de Ciências, tais como a Física, entre os países participantes da Organização para a Cooperação e Desenvolvimento Econômico.

Por meio dos resultados apresentados neste artigo foi possível constatar o potencial pedagógico dos recursos educacionais aumentados em dispositivos móveis, especialmente no uso de recursos multimídia interativos, por possibilitarem que os participantes alterarem os parâmetros das experiências simuladas e verifiquem os resultados de suas próprias ações. No cenário referente ao processo de ensino e aprendizagem de Física, essas operações contribuem tanto para a visualização dos fenômenos físicos, que muitas vezes são invisíveis e/ou difíceis de serem reproduzidos no mundo real, como também para a validação de hipóteses durante as interações dos usuários.

Nesta perspectiva, é importante destacar que a escolha do tipo de recurso educacional é fundamental para alcançar um resultado positivo. Dado que o aplicativo avatAR UFRGS proporcionou experiências educacionais interativas aos usuários, tais como simulações, recursos multimídia (e.g. objetos 3D, vídeos, imagens, e informações contextualizadas), armazenamento dos experimentos no próprio inventário do usuário, além da possibilidade de acesso aos experimentos sem a necessidade de se conectar à Internet. Outra característica observada consiste na baixa granularidade das informações, oferecendo assim, maior nível de detalhamento ao usuário, sendo o conteúdo apresentado em etapas e o usuário podendo interagir com os diferentes níveis de conhecimento para explorar cada conteúdo.

Como trabalhos futuros, esta pesquisa pretende promover as experiências educacionais durante um maior período de tempo com os participantes, a fim de evidenciar se os recursos educacionais aumentados podem contribuir ainda mais para o desenvolvimento de conhecimentos em Física dos usuários. Também pretende-se investigar quais características das interações dos usuários foram mais significativas no desenvolvimento 
VIII Congresso Brasileiro de Informática na Educação (CBIE 2019)

Anais dos Workshops do VIII Congresso Brasileiro de Informática na Educação (WCBIE 2019)

de conhecimentos em Física.

\section{Agradecimentos}

Esta pesquisa é apoiada pela Coordenação de Aperfeiçoamento de Pessoal de Nível Superior (CAPES) e Conselho Nacional de Desenvolvimento Científico e Tecnológico $(\mathrm{CNPq})$, entidades do governo brasileiro focadas no desenvolvimento científico.

\section{Referências}

AVATAR (2019). Página oficial do Projeto AVATAR (Ambiente Virtual de Aprendizagem e Trabalho Acadêmico Remoto). Disponível em: http://www.ufrgs.br/avatar.

Becker, S. A., Cummins, M., Davis, A., Freeman, A., Giesinger, C. H., and Ananthanarayanan, V. (2017). NMC Horizon Report: 2017 Higher Education Edition. Technical report, The New Media Consortium, Austin, Texas.

Billinghurst, M. and Dünser, A. (2012). Augmented reality in the classroom. Computer, 45(7):56-63.

Cai, S., Wang, X., and Chiang, F. K. (2014). A case study of Augmented Reality simulation system application in a chemistry course. Computers in Human Behavior, $37: 31-40$.

Daineko, Y., Dmitriyev, V., and Ipalakova, M. (2017). Using Virtual Laboratories in Teaching Natural Sciences: An Example of Physics Courses in University. Computer Applications in Engineering Education, 25(1):39-47.

Herpich, F. (2019). avatAR UFRGS - Manual do Aplicativo de Realidade Aumentada do Projeto AVATAR. Mendeley Data. Disponível em: http://dx.doi.org/10.17632/w92tdmccvs.3.

Herpich, F., Guarese, R. L. M., Cassola, A. T., and Tarouco, L. M. R. (2018). Realidade Aumentada no Desenvolvimento da Habilidade de Visualização Espacial de Estudantes em Física. VII Congresso Brasileiro de Informática na Educação (CBIE 2018), pages 345-348.

Herpich, F., Nunes, F. B., Petri, G., and Tarouco, L. M. R. (2019). How Mobile Augmented Reality is applied in Education? A systematic literature review. Creative Education, 10(7):1-39.

Ibáñez, M.-B. and Delgado-Kloos, C. (2018). Augmented reality for STEM learning: A systematic review. Computers \& Education, 123:109-123.

Leopold, A. T., Ratcheva, V. S., and Zahidi, S. (2018). The Future of Jobs Report 2018 Insight Report Centre for the New Economy and Society. World Economic Forum.

Nincarean, D., Ali, M. B., Dayana, N., Halim, A., Hishamuddin, M., and Rahman, A. (2013). Mobile Augmented Reality: the potential for education. Procedia - Social and Behavioral Sciences (13th International Educational Technology Conference), 103:657-664.

OECD (2016). PISA 2015 Results (Volume 1): Excellence and Equity in Education. OECD Publishing, Paris. 